\title{
Biomarker-Based Guideline-Directed Medical Therapy of Heart Failure: The Gap Between Guidelines and Clinical Practice
}

\author{
Authors: \\ *Alexander E. Berezin, ${ }^{*}$ Alexander A. Berezin ${ }^{2}$ \\ 1. Department of Internal Medicine, State Medical University, Zaporozhye, Ukraine. \\ 2. Department of Internal Medicine, Medical Academy of Post-Graduate Education, \\ Zaporozhye, Ukraine \\ *Correspondence to aeberezin@gmail.com
}

Disclosure: The authors have declared no conflicts of interest.

Received:

Accepted:

Keywords:

Citation:
20.01.21

14.04.21

Biomarkers, clinical outcomes, heart failure, prediction, risk stratification.

EMJ Cardiol. 2021;9[1]:67-76.

\section{Abstract}

Current clinical recommendations provided by the 2016 European Society of Cardiology (ESC) and 2017 American College of Cardiology (ACC)/American Heart Association (AHA)/Heart Failure Society of America (HFSA) are substantially distinguished in the use of circulating biomarkers in the management of heart failure (HF). To date, natriuretic peptides continue being the universal biomarkers used in diagnosis, risk stratification, and prediction of cardiovascular death, all-cause mortality, and HF-related outcomes for patients with both phenotypes of HF. However, biomarkers of fibrosis and inflammation, including soluble suppressor of tumourgenicity 2 and galectin-3, were able to increase predictive ability of natriuretic peptides in HF patients regardless of cardiovascular riskfactor presentation and HF phenotypes. Therefore, there are many various biomarkers describing several pathophysiological processes such as fibrosis, inflammation, oxidative stress, neurohumoral activation, extracellular matrix turnover, and vascular reparation, that play a pivotal role in the natural evolution of HF. This review discusses whether multiple biomarker models are more effective than a single biomarker in improving risk stratification strategies in patients with HF. It emphasises how in routine clinical practice, the multiple biomarker approach to elicit response to therapy of HF and predict clinical outcomes is rare, probably because of the relatively high cost, low affordability, lack of clear recommendations for clinical implementation, and significant disagreements in the interpretation of the data obtained.

\section{INTRODUCTION}

The prevalence of heart failure (HF) worldwide is 64.34 million cases, and almost 10 million years have been lost due to HF-related disability. ${ }^{1}$ The global trend prevalence of HF with reduced ejection fraction (HFrEF) and preserved ejection

fraction (HFpEF) over the last decade has shown an increase in HFpEF and tendency to stabilisation of newly diagnosed cases of HFrEF. ${ }^{2}$ However, the prevalence of HFrEF in developed countries is decreasing, whereas in developing countries it demonstrated steady growth. ${ }^{3}$ In fact, by the year 2030, in low-to-middle income regions, the prevalence of $\mathrm{HF}$ is estimated to rise 
by over $50 \%$, while high-income countries will have a declined rate of up to $27 \% .{ }^{4}$ Mortality is considerable variable in different regions, i.e., from 34\% in Africa to $7 \%$ in China, with the overall mortality rate being $16.5 \% .^{5}$ This high variability of $\mathrm{HF}$ prevalence and mortality in distinct regions is linked to substantial difference in cardiovascular (CV) risk-factor distribution, affordability of novel technologies in therapy of CV diseases, structure of public health systems, and other factors. ${ }^{5}$

In addition, the presenting clinical syndromes in HFpEF and HFrEF are not distinguishable from one another, but mortality and co-existing comorbidities substantially differ. In this context, the profile of biomarkers that could be used for diagnosis and prognosis of HFpEF would not resemble their signature in HFrEF. Indeed, predictive ability of natriuretic peptides (NPs) in HFrEF was found as higher than in HFpEF. In contrast, soluble suppressor of tumourgenicity 2 (sST2) and galectin-3 when being added to NPs sufficiently improved final discriminative potency of the model in patients with HFpEF. ${ }^{6}$

However, there is no agreement in biomarker profile between the American Heart Association/ American College of Cardiology (AHA/ACC) and the European Society of Cardiology (ESC), ${ }^{6,7}$ A contemporary conceptual framework for the diagnosis and management of HFrEF and HFpEF provided by the AHA (2017) includes the first generation of biomarkers such as NPs, SST2, highly sensitive troponin (hs-Tn), and galectin-3. ${ }^{6}$ Current 2016 ESC HF guidelines indicate the priority of HF having the highest level of evidence before other biomarkers; the discriminative potency of them requires thorough elucidation. ${ }^{7}$

Reflecting the varying stages of evolving HF, biomarkers can predict its clinical course, short- and long-term prognosis, and risks of all-cause mortality and hospital admission. ${ }^{8}$ An abundant number of new biomarkers, i.e., mid-regional pro-adrenomedullin, cystatin-C, IL-6, growth differential factor-15 (GDF-15), matrix metalloproteinases, collagen turnover biomarkers, osteonectin, and others, are now clinically available, and continuous monitoring of their levels in peripheral blood is promising in the context of receiving additional prognostic and diagnostic information, which can improve guiding treatment strategies of different phenotypes of HF. ${ }^{8}$
The aim of this narrative review is to summarise knowledge about prospective potencies of circulating cardiac biomarkers in HF patients that could be useful for routine clinical practice.

\section{METHODS AND METHODOLOGY}

The bibliographic database of life science and biomedical information MEDLINE, EMBASE, Medline (PubMed), the Web of Science, and the Cochrane Central were searched for English publications satisfying the keywords of this study. The authors used the following keywords: "heart failure," "heart failure with reduced ejection fraction," "heart failure with preserved ejection fraction," "cardiac cachexia," "cardiac myopathy," "cardiovascular risk," "cardiovascular risk factors," "cardiac biomarkers," "circulating biomarkers," "secretomics," and "prognosis". Both authors independently evaluated the quality of the articles, correspondence to the main idea of the study, and constructed the final list of the references.

\section{BIOMARKERS FOR HEART FAILURE}

As $\mathrm{HF}$ is a multiply complex disease with sophisticated pathogenesis, which is substantially different for HFrEF and HFpEF, there are many biomarkers proposed to determine stages and severity of the disease, predict occurrence, clinical course and outcomes of HFrEF/HFpEF, a risk of CV and HFrelated mortality, and a response to treatment (Table 1). Previously, there were suggestions that pro-inflammatory conditions, adiposetissue dysfunction, oxidative stress and fibrosis predominantly underlie the pathophysiology of HFpEF, whereas neurohumoral activation, cardiac injury, and biomechanical stress much better describe the adverse cardiac remodelling and natural evolution of HFrEF than HFpEF.,10 This view has been disregarded more recently because biomarkers of biomechanical stress, such as NPs, exhibited their high potency to exclude HFpEF and predict outcomes in both HFrEF and HFpEF, and biomarkers of fibrosis and inflammation, including sST2, galectin-3, increased predictive ability of NPs in $\mathrm{HF}$ patients, regardless of $\mathrm{CV}$ risk-factor presentation and HF phenotypes. .112 $^{12}$ 
Table 1. 2016 European Society of Cardiology (ESC) and 2017 American College of Cardiology (ACC)/American Heart Association (AHA)/Heart Failure Society of America (HFSA) clinical practice guideline recommendations for the use of biomarkers in the management of heart failure.

\begin{tabular}{|c|c|c|c|c|c|c|c|}
\hline \multirow[t]{2}{*}{ Strategy for use } & \multirow[t]{2}{*}{ Biomarkers } & \multicolumn{3}{|c|}{ ESC; 2016} & \multicolumn{3}{|c|}{ ACC/AHA/HFSA; 2017} \\
\hline & & COR & LOE & $\begin{array}{l}\text { Phenotype of } \\
\text { HF }\end{array}$ & COR & LOE & Phenotype of HF \\
\hline Diagnosis & $\begin{array}{l}\text { BNP/NT- } \\
\text { proBNP/MR- } \\
\text { proANP* }\end{array}$ & 1 & A & $\begin{array}{l}\text { AHF, HFpEF, } \\
\text { HFmrEF }\end{array}$ & 1 & A & AHF, CHF \\
\hline \multirow{2}{*}{$\begin{array}{l}\text { Risk of in-hospital } \\
\text { death }\end{array}$} & BNP/NT-proBNP & 1 & C & $\mathrm{AHF}$ & 1 & A & AHF, CHF \\
\hline & hs- TnT/l & I & C & AHF & 1 & A & $\mathrm{AHF}, \mathrm{CHF}$ \\
\hline $\begin{array}{l}\text { Risk of recurrent } \\
\text { hospital } \\
\text { admission }\end{array}$ & BNP/NT-proBNP & \multicolumn{3}{|l|}{ NA } & 1 & A & AHF, CHF \\
\hline \multirow{4}{*}{$\begin{array}{l}\text { Risk of post- } \\
\text { discharged death }\end{array}$} & BNP/NT-proBNP & 1 & A & AHF, CHF & 1 & A & $\mathrm{AHF}, \mathrm{CHF}$ \\
\hline & hs- TnT/l & 1 & C & $\mathrm{AHF}, \mathrm{CHF}$ & I & Ila & $\mathrm{AHF}, \mathrm{CHF}$ \\
\hline & Galectin-3 & \multicolumn{3}{|l|}{ NA } & $11 \mathrm{~b}$ & B & $\mathrm{AHF}, \mathrm{CHF}$ \\
\hline & sST2 & \multicolumn{3}{|l|}{ NA } & $11 \mathrm{~b}$ & B & AHF, CHF \\
\hline $\begin{array}{l}\text { Prevention of HF } \\
\text { onset }\end{array}$ & BNP/NT-proBNP & \multicolumn{3}{|l|}{ NA } & Ila & $B$ & AHF, CHF \\
\hline Guided therapy & BNP/NT-proBNP & \multicolumn{3}{|l|}{ NA } & I & A & HFrEF/HFpEF \\
\hline
\end{tabular}

*Provided for 2016 ESC recommendation only.

ACC: American College of Cardiology; AHA: American Heart Association; AHF: acute heart failure; BNP: B-type natriuretic peptide; CHF: chronic heart failure; COR: class of recommendation; ESC: European Society of Cardiology; HF: heart failure; HFmrEF: heart failure mid-range ejection fraction; HFpEF: heart failure preserved ejection fraction; HFrEF: heart failure reduced ejection fraction; HFSA: Heart Failure Society of America; hs-TnT : high sensitivity troponin; LOE: level of evidence; MR-proANP: mid-regional pro A-type natriuretic peptide; NA: not applicable; NTproBNP: N-terminal pro-B-type natriuretic peptide; SST2: soluble suppressor of tumourgenicity 2.

However, NPs remain universal biomarkers that represent diagnosis, risk stratification and prediction of $\mathrm{CV}$ death, all-cause mortality and HF-related outcomes for patients with both phenotypes of HF.13,14 There are recommendations for use of the levels of B-type NP (BNP) >35 $\mathrm{pg} / \mathrm{mL}$ and N-terminal pro-BNP (NT-proBNP) $>125 \mathrm{pg} / \mathrm{mL}$ to exclude HF in the acute settings. Higher values of these markers (BNP >100 $\mathrm{pg} / \mathrm{mL}$, NT-proBNP $>300 \mathrm{pg} / \mathrm{mL}$, and midregional pro A-type NP [MR-proANP] $>120$ $\mathrm{pmol} / \mathrm{L}$ ) are approved for use in diagnosis of chronic HF., ${ }^{6,7}$ These diagnostic cut-off points are applied strictly, similar to HFrEF and HFpEF, but circulating levels of BNP/NT-proBNP and MR-proANP are frequently lower for patients having HFpEF when compared with those who have HFrEF. A peak value of NT-proBNP $>5,000$ 
$\mathrm{pg} / \mathrm{mL}$ predicts an adverse outcome in HFrEF in-patients, whereas poor prognosis for stable outpatients having HFrEF was suspected when NT-proBNP levels were $>1,000 \mathrm{pg} / \mathrm{mL}$. The trend for reducing the levels of NT-proBNP $>1,000$ $\mathrm{pg} / \mathrm{mL}$ is now considered a concise indicator of adequate therapy of HFrEF patients, while stability of soaring NT-proBNP levels predicts poor clinical outcomes of the disease. ${ }^{14}$ Overall, high levels of NT-proBNP provided similar predictive information in patients with HFpEF as in those with HF mid-range EF (HFmrEF) and HFrEF; other biomarkers, including SST2, galectin-3, and high-sensitivity C-reactive protein (hs-CRP), have demonstrated controversial evidence regarding their ability to be carefully tailored to HF phenotypes and comorbidities. ${ }^{15,16}$ However, negative diagnostic and predictive values of NPs were found to be higher than positive values for prognostication. This implies that single or serial measures of NPs are not enough to completely predict HF evolution, especially among older patients and those who have various $\mathrm{CV}$ diseases and comorbidities. These facts are extremely important because there is no descriptive clinical model that can independently predict the clinical endpoint in HFpEF. ${ }^{17}$

Previous clinical studies have shown that the levels of SST2, galectin-3, and hs-CRP were significantly higher in HFrEF patients when compared with HFpEF individuals. ${ }^{18-21}$ Although peak levels of SST2, high sensitivity troponin (hs$\mathrm{TnT}$ )/I, galectin-3, and hs-CRP, and their dynamic changes substantially improved predictive potency of NPs among patients with HF, there are serious economic concerns regarding the increased number of biomarkers involved in the multiple diagnostic models. ${ }^{22-27}$ In addition, there are sustentative disagreements in justifying whether these biomarkers can be adequately discriminative, and have certain calibration, abilities for reclassification, and likelihood analyses in various cohorts of HF individuals, depending on conventional CV risk factors (sex, age, ischaemic versus non-ischaemic aetiology, left-ventricular EF, estimated glomerular filtration rate, hypertension, and dyslipidaemia), comorbidities (diabetes mellitus, abdominal obesity), New York Heart Association (NYHA) functional class, HF medical therapy, and NTproBNP levels. ${ }^{28}$ In fact, SST2 and galectin-3 not only predicted all-cause and CV death and HF hospitalisation in both HFrEF and HFpEF, with good performance in Kaplan-Meier analysis in face-to-face comparisons with NT-proBNP and hs-TnT/l, but they yielded significant improvement of comparator models (NT-proBNP and hs-TnT/I) adding prognostic information. ${ }^{29-31}$ Consequently, SST2 and galectin-3 have been considered as part of a multiple biomarker panel together with NT-proBNP and hs-TnT for most population HF subgroups independently of comorbidity status and CV risk.

\section{CURRENT CLINICAL RECOMMENDATIONS FOR BIOMARKER UTILITY IN HEART FAILURE}

Current clinical recommendations provided by ESC (2016) and ACC/AHA/Heart Failure Society of America (HFSA; 2017) are substantially distinguished in the use of circulating biomarkers in the management of HF (Table 1). Both clinical guidelines agreed with the diagnostic strategy of acute and chronic HF based on a measure of circulating levels of NPs additionally to clinical signs and symptoms assay, echocardiographic parameter evaluation and analysis of ECG findings. Therefore, the risk of in-hospital death can also be predicted with NT-proBNP peak level at admission and based on a trend of NTproBNP level change. However, other utilities of NPs, such as guided therapy and assay of the risk of recurrent hospital admission, were approved in the 2017 ACC/AHA/HFSA clinical guideline, but not in the 2016 ESC HF recommendation. Moreover, sST2 and galectin-3 as alternative biomarkers for additional risk stratification were recommended by the only 2017 ACC/AHA/HFSA clinical guideline, while the evidence for SST2 and galectin-3 remains very weak, as shown by the low grade of evidence, which currently discourages translation to clinical practice.

Thus, the 2017 ACC/AHA/HFSA clinical practice guideline recommends more extensive biomarker strategy for HF management than the 2016 ESC HF recommendation, while there is a gap of evidence regarding use of biomarkers to predict occurrence of different phenotypes of HF, and limited data for risk stratification depending on CV diseases and comorbidities. 


\section{BIOMARKER PROFILE AND HEART FAILURE STATUS}

There are numerous investigations focusing on the inter-relation between specific biomarkers of fibrosis, inflammation, oxidative stress, neurohumoral activation, extracellular matrix turnover, vascular reparation, and HF status (HFrEF versus HFpEF). ${ }^{32-38}$ Usually, authors executed univariable and multivariable interactions of baseline biomarker levels and outcomes in HF patients with further correction for the $\mathrm{COACH}$ risk engine that included variable anthropometric data, CV diseases (atrial fibrillation, peripheral artery disease, coronary artery disease, dilated cardiomyopathy) and comorbidities (diabetes mellitus, abdominal obesity), estimated glomerular filtration rate, and network analysis. As a result of these investigations, the profile of biomarkers that fitted into HF status for the best was received. ${ }^{37,38}$ The profile of biomarkers might look like this (Figure 1).

Indeed, various circulating biomarkers in key pathophysiological domains are predictive of outcomes in HFpEF and HFrEF, while their circulating levels were substantially different in patients having distinct HF status. For instance, patients with HFrEF had higher median levels of GDF-15, hs-TnT, heart-type fatty-acid-binding protein, and NT-proBNP, but not sST2, galectin-3, hs-CRP, procollagen peptides, and other abundant biomarkers of extracellular turnover, than those who have HFpEF. 21,30,31,39 In contrast, most biomarkers of fibrosis, inflammation and extracellular matrix remodelling have demonstrated higher levels in HFpEF than HFrEF. ${ }^{21,26,30-33,38}$ A novel paradigm for HFpEF has been described in close connection with $\mathrm{CV}$ risk factors and comorbidities, which drive microvascular inflammation, endothelial dysfunction, altered vascular repair, adverse cardiac remodelling, and dysfunction of skeletal muscles and adipose tissue. ${ }^{40}$ In fact, the presentation and number of risk factors contributing to HFpEF distinguish from those that correspond with HFrEF and demonstrate strong association with age, sex, genetic predisposition, ethnicity, level of education, and region. ${ }^{41,42}$ In this context, dominant biomarker clusters are required to thoroughly identify individuals at risk of HF occurrence and mortality due to cardiac dysfunction. Consequently, multiple biomarker models appear to be more prognostic than single biomarkers in risk stratification strategies in patients at high risk of HFpEF and those who have overt HFpEF. Obviously, the optimal choice of biomarker combination is strongly needed for effective multiple biomarker strategy to improve HF patient management and outcomes. However, there are limited data to conclude whether multiple biomarker models are better in HFpEF when compared with HFrEF to predict HF-related outcomes and death. ${ }^{88,39}$

\section{MULTIPLE BIOMARKER MODELS}

The panel of biomarkers, which measures diverse biological processes and can be a prognostic tool in HFrEF, was investigated by Ky B et al. ${ }^{43}$ In a multicentre cohort of 1,513 patients with HFrEF, the levels of several biomarkers such as hs-CRP, myeloperoxidase, BNP, soluble FMS-like tyrosinekinase receptor-1, Tn-1, soluble toll-like receptor-2, creatinine, and uric acid were measured. Authors created multiple biomarker scores and assessed their performance for prediction of the risk of death, cardiac transplantation, or as a ventricularassistant device in comparison with conventional clinical risk scores (the Seattle Heart Failure Model [SHFM]) for 2.5 years. Investigators found that patients with HFrEF have the highest tertile of the multiple biomarker score had a 13.7-fold increased risk of adverse clinical outcomes when compared with those who had the lowest tertile (95\% confidence interval [Cl]: 8.75-21.50). Moreover, these effects were independent of the SHFM and adding the multiple biomarker score to the SHFM markedly improved its discriminative potency.

To characterise HF status, and evaluate a possible relationship between $\mathrm{HF}$ status and the risk of all-cause death or HF-related hospital admissions, Chirinos et al. ${ }^{44}$ used 49 plasma biomarkers received from patients withn HFpEF $(n=379)$ enrolled in the TOPCAT trial. The authors constructed several clusters, which included biomarkers of fibrosis/tissue remodelling (sST2), inflammation (TNF-a, soluble TNF-receptor 1, and IL-6), renal injury/dysfunction (cystatin-C), liver fibrosis (YKL-40), neurohormonal regulators of mineral metabolism/calcification (FGF-23 and osteoprotegerin), intermediary metabolism/ adipose-tissue dysfunction (fatty-acid- 
- HSP-60

- strail

- Pentraxin-3

- H-FABR

- NGAL

- Cystatin-C

- TNF-a

- Myostatin

- GDF-15

- hs-TnT/l

- Copeptin

- MR-pro-

adrenomedullin

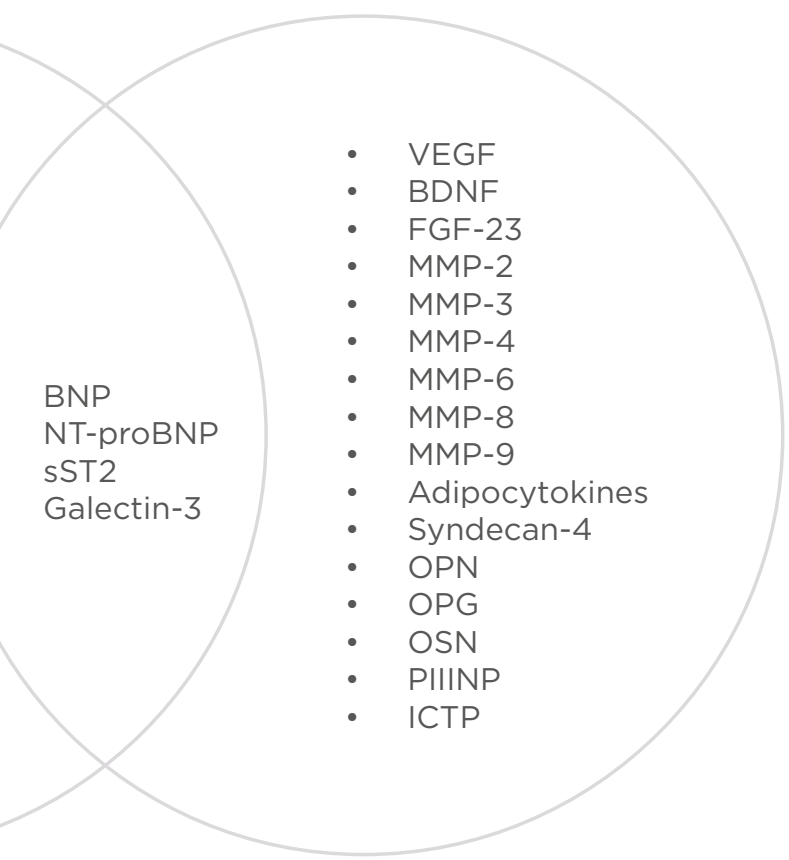

Figure 1: Corresponding biomarker profiles with heart failure status.

BDNF: Brain-derived neurotrophic factor; BNP: B-type natriuretic peptide; FGF-23: fibroblast growth-factor-23; GDF15: growth differential factor-15; H-FABP: heart-type fatty-acid-binding protein; ICTP: collagen type I carboxy-terminal telopeptide; MMP: matrix metalloproteinase; MR-proANP: mid-regional pro A-type natriuretic peptide; NGAL: neutrophil gelatinase-associated lipocalin; NT-proBNP: N-terminal pro-B-type natriuretic peptide; PIIINP: procollagen type III N-terminal propeptide; sST2: soluble suppressor of tumourgenicity 2; VEGF: vascular endothelial growth factor.

binding protein-4 and GDF-15), angiogenesis (angiopoietin-2), biomarkers of myocardial injury (hs-TnT), extracellular matrix remodelling (MMP-7), and biomechanical stress (NT-proBNP). Using a machine-learning-derived model, the authors found that a combination of biomarkers was strongly predictive of the risk of HF-related hospital admission and sufficiently improved the risk prediction when added to the MetaAnalysis Global Group in Chronic Heart Failure (MAGGIC) risk score. ${ }^{44}$ In addition, the model markedly predicted the risk of admission due to HF progression (hazard ratio: 2.74 ; 95\% Cl: $1.93-$ 3.90; $p<0.0001$ ), which was also independent of the MAGGIC risk score. ${ }^{44}$

Using a random algorithm Yuan et al. ${ }^{46}$ found that the combination of creatine kinase-MB, BNP, galectin-3, and SST2 were useful for prediction of HFpEF/HFrEF occurrence. ${ }^{45}$ In contrast, Zhang et al. $^{46}$ reported that discriminative ability of sST2 and NT-proBNP for 1-year all-cause death in patients with acute HF was similar and remained significant between patients having ischaemic and non-ischaemic aetiology of HF.
However, galectin-3 did not increase prognostic ability of SST2 and NT-proBNP when added to this combination in patients with ischaemic HFrEF, but not in individuals with non-ischaemic HF regardless of its phenotype. In a cohort of adult patients with HF due to congenital heart disease, a multiple biomarker model constructed from neurohormones (angiotensin II, endothelin-1, norepinephrine, aldosterone, and plasma renin activity), inflammatory biomarkers (hs-CRP, hsTNF, soluble TNF receptor Types I and II, and IL-6), and BNP, predicted HF mortality. ${ }^{47}$ Thus, aetiology of acute HF should be considered when an optimal panel of biomarkers has been validated.

Jackson et al. ${ }^{48}$ measured the levels of biomarkers received from 628 inpatients with acutely decompensated HF. The authors noticed that patients did not always have markedly increased circulating levels of mid-regional proadrenomedullin, MR-proANP, copeptin, hs-cTnT, sST2, galectin-3, cystatin-C, combined free light chains, and hs-CRP. Consequently, authors undertook a dichotomisation into low (up to 
two elevated biomarkers) or high (at least three and more elevated biomarkers) risk groups. It was found that patients with HF from the highrisk group provided much more incremental prognostic value than individuals from the lowrisk group (hazard ratio: 2.20; 95\% Cl: 1.37-3.54; $\mathrm{p}=0.001$ ). Finally, elevated circulating levels of five biomarkers demonstrated the highest predictive ability for the risk of death. ${ }^{48}$

Interestingly, in the patient population with HF $(n=1,497)$ enrolled in the CORONA study, a multiple biomarker approach using two panels of biomarkers, which included model 1 (endostatin, IL-8, sST2, TnT, galectin-3, and C-C motif ligand 21) and model 2 (TnT, sST2, galectin-3, pentraxin-3, and soluble TNF-receptor-2), in addition to hs-CRP and NT-proBNP, demonstrated limited attributive potency of inflammatory biomarker panels for identifying the risk of adverse clinical outcomes. ${ }^{49}$ Therefore, in the PLATO study, elevated baseline levels of NT-proBNP and GDF-15 were strong predictors for all-cause death based on their associations with HF-related death, as well as arrhythmia and sudden cardiac death among patients with acute coronary syndrome. ${ }^{50}$ Unfortunately, in routine clinical practice, a multiple biomarker approach to elicit response to HF therapy and predict clinical outcomes is very rare, probably due to the relatively high cost, low affordability, lack of clear recommendations for clinical implementation, and significant disagreements in the interpretation of data obtained.

\section{GUIDED THERAPY OF HEART FAILURE}

While the current data on using biomarkers to guide HF management remain mixed, more research is necessary to better understand how to utilise biomarkers to improve HF management. ${ }^{51}$ Guided therapy in clinical practice is mostly based on serial changes of NPs, while other biomarkers such as SST 2 and galectin- 3 have been considered as a component of this strategy. ${ }^{52}$ Previous proofof-concept studies have reported controversial results for biomarker-guided strategies in HF. ${ }^{53}$ The GUIDE-IT study did not find benefit from biomarker-guided therapy versus usual care in improving the primary endpoints of HF hospital admission or CV mortality in patients with overt HF. ${ }^{54}$ However, HFrEF patients whose NTproBNP levels decreased to $\leq 1,000 \mathrm{pg} / \mathrm{mL}$ over
90 days of HF therapy had better outcomes and significantly better quality of life than those who had no reduced levels of the biomarker. ${ }^{55}$ These findings urge us to reassume whether guide therapy is a powerful tool for the entire population of HF patients. However, the role of a multiple-biomarker strategy in guided therapy is not certain, and well-designed, largescale, multicentre, randomised clinical trials are definitively required to shed light on these approaches to HF management.

\section{COST/BENEFIT TO USE OF BIOMARKERS}

Implementation of biomarker strategies in routine clinical practice corresponds to substantial cost for patients and the health system. Consequently, biomarker-guided decisions should desirably yield economic benefit in HF administration and a better allocation of financial resources. Indeed, the REACH-HF trial has shown that home-based facilitated intervention (with inclusion of predominantly NP biomarkers) for HFrEF was clinically superior in disease-specific 1-year quality of life and, thereby, offers an affordable alternative to traditional centre-based programmes for HFrEF. ${ }^{56}$ In addition, the risk stratification of elderly patients with HF, based on multiparametric approach, ensured cost benefit in quality of life. ${ }^{57}$ However, the 2016 ESC and 2017 AHA/ACC guidelines did not concisely emphasise which patients with HF might especially benefit from the biomarker approach. It is reasonable to consider the administration of NPs in patients with HFrEF/HFpEF, while other biomarkers (cardiac Tns, sST2, galectin-3) could have a significant economic impact in predicting all-cause mortality, identifying new patients with HF requiring hospitalisation, optimising treatment, and consequently preserving hospital budget. ${ }^{39,58-60}$ In fact, uncertain cost/benefit ratio is still one of the unsolved problems for a non-NP biomarker strategy for an unselected real-world population. This is the reason most biomarker models currently cannot be applied in routine clinical practice.

\section{PROSPECTIVES}

Several underlying pathophysiological processes (extracellular matrix structural constituents, 
proteinaceous extracellular matrix) and signalling pathways (regulation of apoptotic process and integrin signalling pathway) involved in the pathogenesis of HFrEF/HFpEF can be described by the signature of non-coding RNAs. Indeed, transcriptome analysis offers great potential in identifying HF biomarkers. Among 1,139 differentially expressed messenger RNAs, He et al. ${ }^{61}$ identified clusters constructed from nine long non-coding RNAs, three micro-RNAs, and 25 messenger RNAs that were closely associated with progression and outcomes of HF. However, the predictive value of the RNA signature requires elucidation in large clinical trials. ${ }^{62}$ However, metabolomic and lipidomic phenotyping of patients having HFrEF/HFpEF to indicate a profile of oxidative stress, lactic acidosis, and metabolic syndrome, coupled with mitochondria dysfunction, is a promising approach to stratify them at high risk of poor outcomes. ${ }^{63}$

\section{CONCLUSION}

In conclusion, cardiac biomarkers such as NPs are a promising tool for individualising care in patients with HF, whereas alternative biomarkers (sST2, hs-TnT/l, galectin-3) having weaker clinical evidence than NPs and require more investigation to easily identify the target population in which they would have most cost benefit. A multiple biomarker approach is probably more optimistic for HFrisk stratification and predicting HF-related outcomes than a single biomarker approach, especially in patients with older age, HFpEF, and those having comorbidities. Optimal choice of biomarkers for panels requires in-depth evaluation of economic burden, and not only their discriminative probability for all-cause and CV mortality, HF occurrence, and HF incidence. New biomarkers of the inflammatory axis, matrix remodelling, fibrosis, metabolic axis, and oxidative stress demonstrate uncertainty in their potential therapeutic interventions and are under follow-up investigation. Large clinical trials are required to better understand the role of a multiple-biomarker strategy in HF care to decrease morbidity and mortality, improve quality of life, and propose an easy-to-execute approach for routine clinical practice.

\section{References}

1. Lippi G, Sanchis-Gomar F. Global epidemiology and future trends of heart failure. AME Med J. 2020;5:15.

2. Benjamin EJ et al.; American Heart Association Council on Epidemiology and Prevention Statistics Committee and Stroke Statistics Subcommittee. Heart disease and stroke statistics-2019 update: a report from the American Heart Association. Circulation. 2019;139(10):e56-528.

3. Conrad $\mathrm{N}$ et al. Temporal trends and patterns in heart failure incidence: a population-based study of 4 million individuals. Lancet. 2018;391(10120):572-80.

4. GBD 2017 Disease and Injury Incidence and Prevalence Collaborators. Global, regional, and national incidence, prevalence, and years lived with disability for 354 diseases and injuries for 195 countries and territories, 1990-2017: a systematic analysis for the Global Burden of Disease Study 2017. Lancet. 2018;392(10159):1789-858.

5. Dokainish $\mathrm{H}$ et al.; INTER-CHF Investigators. Global mortality variations in patients with heart failure: results from the International Congestive Heart Failure (INTER-CHF) prospective cohort study. Lancet Glob Health. 2017; 5(7):e665-72.

6. Chow SL et al.; American Heart Association. Role of biomarkers for the prevention, assessment, and management of heart failure: a scientific statement from the American Heart Association. Circulation. 2017;135(22):e1054-91.

7. Ponikowski P et al. 2016 ESC guidelines for the diagnosis and treatment of acute and chronic heart failure: the Task Force for the Diagnosis and Treatment of Acute and Chronic Heart Failure of the European Society of Cardiology (ESC). Developed with the special contribution of the Heart Failure Association (HFA) of the ESC. Eur J Heart Fail. 2016;18(8):891-975.

8. Choudhary $\mathrm{R}$ et al. Heart failure biomarkers. J Cardiovasc Transl Res. 2013;6(4):471-84.

9. Zile MR, Baicu CF. Biomarkers of diastolic dysfunction and myocardial fibrosis: application to heart failure with a preserved ejection fraction. $J$ Cardiovasc Transl Res. 2013;6(4):501-15.

10. van Kimmenade RR, Januzzi JL Jr. Emerging biomarkers in heart failure. Clin Chem. 2012;58(1):127-38.
11. Paul S, Harshaw-Ellis K. Evolving use of biomarkers in the management of heart failure. Cardiol Rev. 2019; 27(3):153-9.

12. Berezin $A E$ et al. Emerging role of adipocyte dysfunction in inducing heart failure among obese patients with prediabetes and known diabetes mellitus. Front Cardiovasc Med. 2020;7:583175.

13. Zheng SL et al. Drug treatment effects on outcomes in heart failure with preserved ejection fraction: a systematic review and meta-analysis. Heart. 2018;104(5):407-15

14. Lam CSP et al. The role of $\mathrm{N}$-terminal pro-B-type natriuretic peptide in prognostic evaluation of heart failure. J Chin Med Assoc. 2019;82(6):447-51.

15. Savarese $\mathrm{G}$ et al. Associations with and prognostic and discriminatory role of $\mathrm{N}$-terminal pro-B-type natriuretic peptide in heart failure with preserved versus mid-range versus reduced ejection fraction. J Card Fail. 2018;24(6):365-74.

16. Shah $\mathrm{KS}$ et al. Heart failure with preserved, borderline, and reduced ejection fraction: 5-year outcomes. J Am Coll Cardiol. 2017;70(20):2476-86. 
17. Löfström $U$ et al. Prognostic impact of Framingham heart failure criteria in heart failure with preserved ejection fraction. ESC Heart Fail. 2019;6(4):830-9.

18. Najjar E et al. ST2 in heart failure with preserved and reduced ejection fraction. Scand Cardiovasc J. 2019;53(1):21-7.

19. Santhanakrishnan R et al. Growth differentiation factor 15, ST2, highsensitivity troponin $\mathrm{T}$, and $\mathrm{N}$-terminal pro brain natriuretic peptide in heart failure with preserved vs. reduced ejection fraction. Eur J Heart Fail. 2012;14(12):1338-47.

20. Sinning $C$ et al. Biomarkers for characterization of heart failure - distinction of heart failure with preserved and reduced ejection fraction. Int J Cardiol. 2017;227:272-7.

21. Mitic VT et al. Cardiac remodeling biomarkers as potential circulating markers of left ventricular hypertrophy in heart failure with preserved ejection fraction. Tohoku J Exp Med. 2020;250(4):233-42.

22. McLellan J et al. Natriuretic peptideguided treatment for heart failure: a systematic review and meta-analysis. BMJ Evid Based Med. 2020;25(1):33-7.

23. Aimo A et al. Prognostic value of soluble suppression of tumorigenicity-2 in chronic heart failure: a meta-analysis. JACC Heart Fail. 2017;5(4):280-6.

24. Chen YS et al. Using the galectin-3 test to predict mortality in heart failure patients: a systematic review and meta-analysis. Biomark Med. 2016;10(3):329-42.

25. Oikonomou E et al. Galectin-3: a pathophysiological background index or an emerging prognostic biomarker in heart failure? J Am Coll Cardiol. 2019;73(14): 1875.

26. Lakhani I et al. Diagnostic and prognostic value of serum C-reactive protein in heart failure with preserved ejection fraction: a systematic review and meta-analysis. Heart Fail Rev. 2020;26(5):1141-50.

27. Aimo A et al. Prognostic value of high-sensitivity troponin $\mathrm{t}$ in chronic heart failure: an individual patient data meta-analysis. Circulation. 2018; 137(3):286-97.

28. Bayes-Genis A, Ordonez-Llanos J. Multiple biomarker strategies for risk stratification in heart failure. Clin Chim Acta. 2015;443:120-5.

29. Emdin M et al. sST2 predicts outcome in chronic heart failure beyond NT-proBNP and high-sensitivity troponin T. J Am Coll Cardiol. 2018:72(19):2309-20.

30. Jirak $P$ et al. Expression of the novel cardiac biomarkers SST2, GDF15, suPAR, and $\mathrm{H}-\mathrm{FABP}$ in HFpEF patients compared to ICM, DCM, and controls. J Clin Med. 2020;9(4):1130.
31. Rabkin SW, Tang JKK. The utility of growth differentiation factor-15, galectin-3, and SST2 as biomarkers for the diagnosis of heart failure with preserved ejection fraction and compared to heart failure with reduced ejection fraction: a systematic review. Heart Fail Rev. 2020;26(4)799-812

32. Agarwal I et al. Fibrosis-related biomarkers and incident cardiovascular disease in older adults: the cardiovascular health study. Circ Arrhyth Electrophysiol. 2014;7:583-9.

33. Wang TJ et al. Clinical and echocardiographic correlates of plasma procollagen type III amino-terminal peptide levels in the community. Am Heart J. 2007;154(2):291-7.

34. Pan W, et al. Comparison of predictive value of NT-proBNP, sST2 and MMPs in heart failure patients with different ejection fractions. BMC Cardiovasc Disord. 2020;20(1):208.

35. Iraqi $W$ et al. Extracellular cardiac matrix biomarkers in patients with acute myocardial infarction complicated by left ventricular dysfunction and heart failure: insights from the Eplerenone PostAcute Myocardial Infarction Heart Failure Efficacy and Survival Study (EPHESUS) study. Circulation. 2009;119(18):2471-9.

36. Berezin $A E$ et al. Altered signature of apoptotic endothelial cell-derived microvesicles predicts chronic heart failure phenotypes. Biomark Med. 2019;13(9):737-50

37. Huang A et al. Prognostic value of sST2 and NT-proBNP at admission in heart failure with preserved, midranged and reduced ejection fraction. Acta Cardiol. 2018;73(1):41-8.

38. Tromp $\mathrm{J}$ et al. Biomarker profiles in heart failure patients with preserved and reduced ejection fraction. J Am Heart Assoc. 2017;6(4):e003989

39. Topf A et al. The diagnostic and therapeutic value of multimarker analysis in heart failure. An approach to biomarker-targeted therapy. Front Cardiovasc Med. 2020;7:579567.

40. Paulus WJ, Tschöpe C. A novel paradigm for heart failure with preserved ejection fraction: comorbidities drive myocardial dysfunction and remodeling through coronary microvascular endothelial inflammation. J Am Coll Cardiol. 2013:62(4):263-71

41. Dunlay SM et al. Epidemiology of heart failure with preserved ejection fraction. Nat Rev Cardiol. 2017;14(10):591-602

42. Lam CSP et al. Sex differences in heart failure. Eur Heart $\mathrm{J}$ 2019;40(47):3859-68c.

43. Ky B et al. Multiple biomarkers for risk prediction in chronic heart failure. Circ Heart Fail. 2012;5(2):183-90.
44. Chirinos JA et al. Multiple plasma biomarkers for risk stratification in patients with heart failure and preserved ejection fraction. J Am Coll Cardiol. 2020;75(11):1281-95

45. Yuan $\mathrm{H}$ et al. Development of heart failure risk prediction models based on a multi-marker approach using random forest algorithms. Chin Med J (Engl). 2019;132(7):819-26.

46. Zhang $M$ et al. Comparison of multiple biomarkers for mortality prediction in patients with acute heart failure of ischemic and nonischemic etiology. Biomarker Med. 2018;12(11):1207-17.

47. Miyamoto $\mathrm{K}$ et al. Prognostic value of multiple biomarkers for cardiovascular mortality in adult congenital heart disease: comparisons of single-/twoventricle physiology, and systemic morphologically right/left ventricles. Heart Vessels. 2016;31(11):1834-47.

48. Jackson CE et al. The incremental prognostic and clinical value of multiple novel biomarkers in heart failure. Eur J Heart Fail. 2016;18(12):1491-8.

49. Nymo SH et al. Limited added value of circulating inflammatory biomarkers in chronic heart failure. JACC Heart Fail. 2017;5(4):256-64.

50. Lindholm D et al. Association of multiple biomarkers with risk of allcause and cause-specific mortality after acute coronary syndromes: a secondary analysis of the PLATO biomarker study. JAMA Cardiol. 2018;3(12):1160-6.

51. Chang $\mathrm{KW}$ et al. Using biomarkers to guide heart failure management. Expert Rev Cardiovasc Ther. 2017;15(10):729-41.

52. Pruett $A E$ et al. Evolution of biomarker guided therapy for heart failure: current concepts and trial evidence. Curr Cardiol Rev. 2015;11(1):80-9.

53. Felker GM et al. Effect of natriuretic peptide-guided therapy on hospitalization or cardiovascular mortality in high-risk patients with heart failure and reduced ejection fraction: a randomized clinical trial. JAMA. 2017;318(8):713-20.

54. Ibrahim NE, Januzzi JL Jr. The future of biomarker-guided therapy for heart failure after the guiding evidence-based therapy using biomarker intensified treatment in heart failure (GUIDE-IT) study. Curr Heart Fail Rep. 2018;15(2):37-43.

55. Januzzi JL Jr et al. Natriuretic peptide response and outcomes in chronic heart failure with reduced ejection fraction. J Am Coll Cardiol. 2019; 74(9):1205-17.

56. Dalal HM et al. The effects and costs of home-based rehabilitation for heart failure with reduced ejection fraction: The REACH-HF multicentre 
randomized controlled trial. Eur J Prev Cardiol. 2019;26(3):262-72.

57. Rosa GM et al. Predictors of cardiovascular outcome and rehospitalization in elderly patients with heart failure. Eur J Clin Invest. 2019;49(2):e13044.

58. Pufulete $M$ et al. Effectiveness and cost-effectiveness of serum B-type natriuretic peptide testing and monitoring in patients with heart failure in primary and secondary care: an evidence synthesis, cohort study and cost-effectiveness model. Health Technol Assess. 2017;21(40):1-150.
59. Clerico A et al. Evidence on clinical relevance of cardiovascular risk evaluation in the general population using cardio-specific biomarkers. Clin Chem Lab Med. 2020;59(1):79-90.

60. Gruson D et al. Measurement of Galectin-3 with the ARCHITECT assay: clinical validity and costeffectiveness in patients with heart failure. Clin Biochem. 2014;47(12):1006-9.

61. $\mathrm{He} Y$ et al. Exploring biomarkers and therapeutic targets for pressure overload induced heart failure based on microarray data. Cardiovasc Diagn
Ther. 2020;10(5):1226-37.

62. Matkovich SJ. Transcriptome analysis in heart failure. Curr Opin Cardiol. 2016;31(3):242-8.

63. Contaifer D Jr et al. Metabolic modulation predicts heart failure tests performance. PLoS One. 2019;14(6):eO218153. 